\title{
MESURE AUTOMATIQUE DU FROTTEMENT INTÉRIEUR ET DU DÉFAUT DE MODULE A L'AIDE D'UN PENDULE DE TORSION INVERSE (*)
}

\author{
B. BAYS et P. A. GRANDCHAMP \\ Laboratoire de Génie Atomique, E. P. F., Lausanne
}

(Reçu le 15 décembre 1969)

\begin{abstract}
Résumé. - Les auteurs décrivent un système de détection optique des oscillations d'un pendule de torsion inversé. Un dispositif électronique mesure de manière entièrement automatique l'amplitude des oscillations du pendule en régime d'amortissement libre, leur période et la température de l'échantillon.

Ces grandeurs sont perforées sur bande télex, le frottement intérieur et le défaut de module sont calculés ensuite en fonction de la température par un ordinateur. Il est également décrit un dispositif électronique à l'aide duquel la valeur du frottement intérieur est enregistrée graphiquement.

Abstract. - The authors describe a system for the optical detection of oscillations of an inverted torsion pendulum. An electronic device measures automatically the amplitude of the pendulum oscillations in free decay, their period and the sample temperature.

These values are punched on a telex tape and then processed by a computer. An electronic device with which the value of internal friction is graphically registered is also described.
\end{abstract}

1. Introduction. - Lorsqu'un échantillon est soumis à une sollicitation mécanique sinusoïdale, il apparaît généralement un déphasage $\delta$ entre la contrainte et la déformation.

On appelle frottement intérieur la grandeur $\operatorname{tg} \delta$ pouvant être calculée à partir de la relation

$$
\operatorname{tg} \delta=\frac{\mathrm{i}}{n \pi} \ln \frac{A_{i}}{A_{i+n}}
$$

où $A_{i}$ et $A_{i+n}$ sont respectivement la $i$-ième et la $(i+n)$-ième amplitude d'oscillation mesurée en amortissement libre (lorsque, par exemple, un fil est soumis à des déformations oscillatoires de torsion au moyen d'un pendule de torsion inversé) [1].

Le défaut de module $\Delta G / G$ est calculé à partir de la relation

$$
\Delta G / G=\left(f_{0}^{2}-f^{2}\right) / f_{0}^{2}
$$

où $f$ et $f_{0}$ représentent la fréquence des oscillations du pendule avec l'échantillon étudié, respectivement avec un échantillon pris comme référence.

Généralement on mesure le frottement intérieur et le défaut de module en fonction de la température et de ce fait ces mesures peuvent s'avérer très longues. Il nous a donc paru utile de développer un dispositif de mesure entièrement automatique.

En fait, le frottement intérieur n'est pas calculé directement à partir de la formule (1) en mesurant simplement deux amplitudes, mais en mesurant la suite des amplitudes $A_{i+k n}$, où $i$ et $n$ sont choisis et

(*) Ce travail a été subventionné par le crédit No 2.60 .68 du Fonds National Suisse pour la Recherche Scientifique. $k$ prend les valeurs entières de 1 à 4 ou de 1 à 8 . Un calcul effectué au moyen d'un ordinateur permet alors d'obtenir le frottement intérieur avec une plus grande précision [2].

2. La détection des oscillations. - Un faisceau lumineux est réfléchi par un miroir solidaire du pendule (Fig. 1). Lorsque le pendule oscille, le rayon réfléchi balaye un angle double de l'angle de torsion du pendule. Ce rayon lumineux est ensuite projeté sur un écran formé de photocellules au silicium du type cellules solaires où il forme un spot rectangulaire. Ces photocellules au nombre de 8 sont assemblées en deux rangées de 4.

L'expérience montre que le courant de court-circuit fourni par une photocellule est proportionnel à la portion de surface éclairée. Comme le courant des quatre photocellules d'une rangée est opposé à celui de l'autre, le courant total fourni par ce montage est proportionnel à la différence des surfaces éclairées de chaque rangeé. Etant donné que l'angle de torsion du pendule est très faible $\left(10^{-5}-10^{-2}\right.$ radiant), le déplacement du spot sur les photocellules peut être considéré comme étant proportionnel à l'angle de torsion du pendule; il en est de même pour le courant fourni par le dispositif décrit ci-dessus. Ceci permet ainsi de mesurer les amplitudes et la fréquence des oscillations du pendule.

Il est fréquent qu'en cours de mesure, l'effet de relâchement des contraintes internes de l'échantillon produise une dérive mécanique du pendule. Nous avons prévu de corriger automatiquement cette dérive par l'intermédiaire d'un miroir qui peut être orienté au moyen d'un moteur (Fig. 1). 


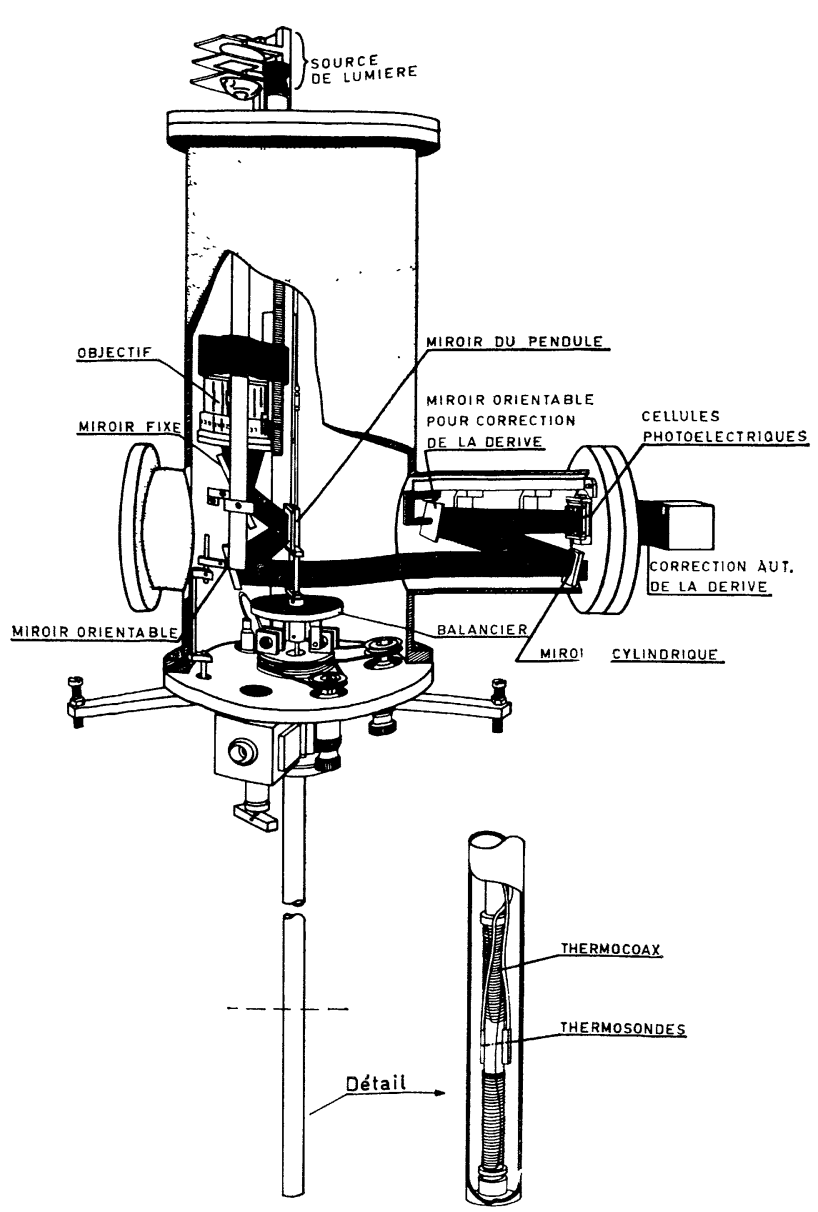

FIG. 1. - Détection des oscillations du pendule, correction de l'effet de la dérive du zéro mécanique, cryostat et thermosondes.

3. Les fonctions du dispositif électronique. - Généralités. - Les signaux analogiques fournis par les cellules photoélectriques, soit l'élongation $a(t) \mathrm{du}$ pendule et les signaux fournis par les thermosondes, soit la température de l'échantillon, sont traités par un dispositif électronique qui doit remplir les fonctions suivantes (Fig. 2):

3.1 fournir un signal digital pour la mesure des amplitudes successives ;

3.2 fournir un signal digital pour la mesure de la période des oscillations ;

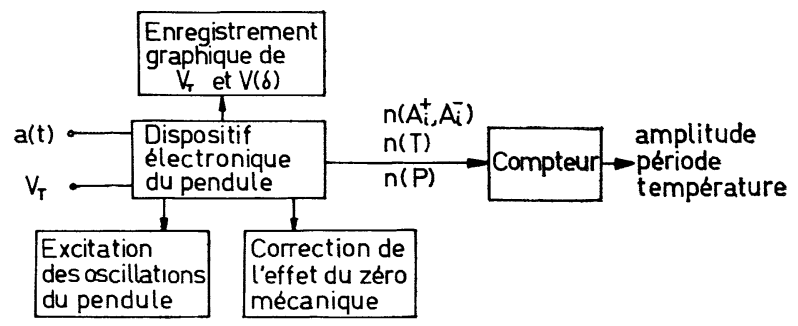

FIG. 2. - Les fonctions du dispositif électronique du pendule : $a(t)$, élongation du pendule; $V_{\mathrm{T}}$, température de l'échantillon; $V(\delta)$, frottement intérieur ; $n\left(A_{i}^{+}, A_{i}^{-}\right)$, valeurs de crête de $a(t)$; $n(\mathrm{~T})$, température de l'échantillon (après conversion en grandeur digitale de $\left.V_{\mathrm{T}}\right) ; n(\mathrm{P})$, période des oscillations du pendule.
3.3 convertir en signal digital la grandeur fournie par les thermosondes ;

3.4 fournir un signal permettant de corriger l'effet de la dérive mécanique du pendule ;

3.5 fournir un signal analogique pour l'enregistrement graphique du frottement intérieur ;

3.6 fournir le courant d'excitation pour l'entretien des oscillations du pendule à l'amplitude désirée, avant la phase de mesure ;

3.7 commander les diverses opérations de mesures, de perforation de celles-ci, ainsi que l'entretien du pendule.

3.1 Mesure digitale des AmPlitudes. - 3.1.1 Le principe. - Le courant fourni par les photocellules est converti en tension puis amplifié. Les valeurs de crête de ce signal, qui est de la forme

$$
a(t)=a_{0} \mathrm{e}^{-\lambda t} \sin (\omega t+\varphi)
$$

en régime d'amortissement libre, sont mesurées par un voltmètre digital composé d'un convertisseur analogique-digital [3] et d'un compteur (Fig. 3). Pour mesurer

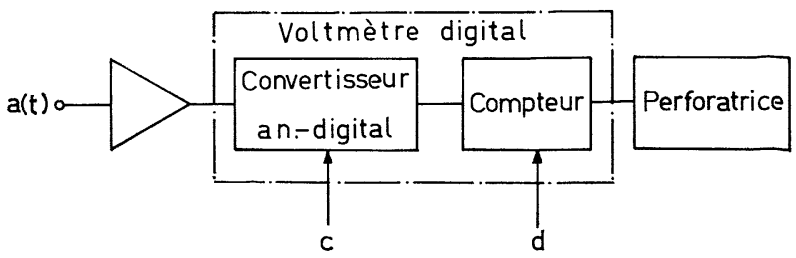

FIG. 3. - Schéma de principe de la mesure des valeurs de crête de $a(t): c$, commande de la mesure des valeurs de crête de $a(t)$; $d$, commande de la perforation de $n\left(A_{i}^{+}, A_{i}^{-}\right)$(valeurs de crête de $a(t)), n(\mathrm{~T})$ (température de l'échantillon) et $n(\mathrm{P})$ (période des oscillations du pendule).

ces valeurs de crête on mesure la tension $a(t)$ à l'instant où elle passe par un extrêmum $A_{i}$. La mesure dure au maximum $5 \mathrm{~ms}$ alors que la fréquence du signal $a(t)$ ne dépasse pas $10 \mathrm{c} / \mathrm{s}$. L'ordre de mesure est donné au voltmètre digital par une impulsion $c$. Pour éviter des erreurs pouvant provenir d'une dérive mécanique du pendule, nous mesurons à la $i$-ième oscillation la valeur de crête positive $A_{i}^{+}$et la valeur de crête $A_{i}^{-}$qui lui succède et on fait la somme :

$$
A_{i}=A_{i}^{+}+A_{i}^{-} \text {. }
$$

Dès que la mesure de $A_{i}^{-}$est exécutée, une impulsion $d$ ordonne la perforation de la valeur $A_{i}(\S 3.7)$.

L'impulsion de commande $c$ est obtenue de la manière suivante (Fig. 4 et $\S 3.1 .3$ ) :

- $a(t)$ est déphasé de $\pi / 2$, ce qui fournit un signal proportionnel à $\dot{a}(t)$; nous avons construit un déphaseur dans la gamme de $10^{-2} \mathrm{c} / \mathrm{s}$ à $10 \mathrm{c} / \mathrm{s}$;

- $\dot{a}(t)$ est écrêté ce qui fournit le signal $b(t)$.

Les fronts montants et descendants de $b(t)$ commandent deux monostables dont la somme des impulsions donne le signal $c$. 


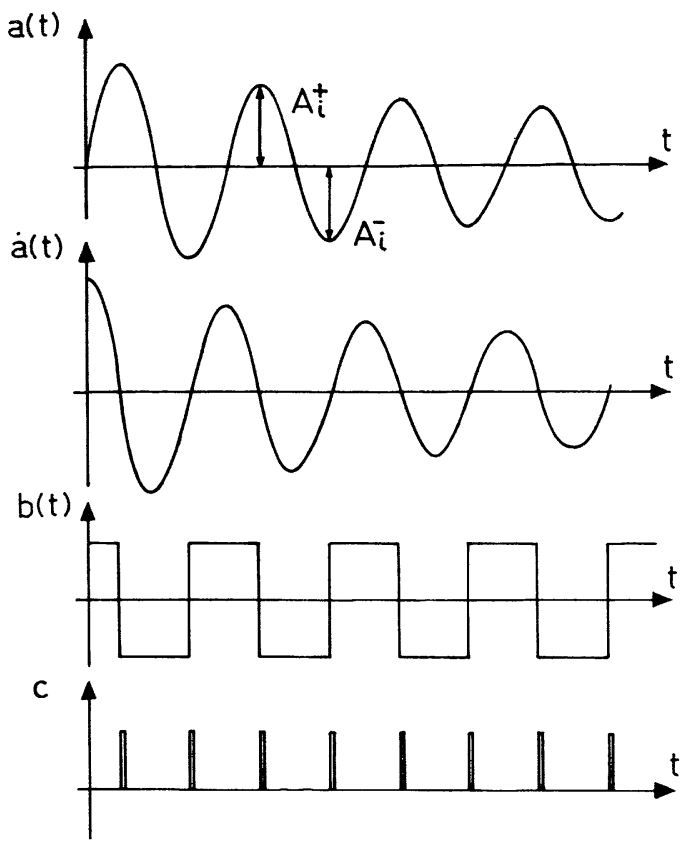

Fig. 4. - Obtention des impulsions $c: a(t)$, signal fourni par les photocellules après conversion en tension; $\dot{a}(t)$, signal $a(t)$ déphasé de $\pi / 2 ; b(t)$, signal $\dot{a}(t)$ écrêté.

3.1.2 Conversion en tension et amplification $d u$ courant fourni par les photocellules. - Le courant délivré par les photocellules traverse une résistance de charge de $10 \Omega$ (Fig. 5). La tension aux bornes de cette résistance $(<20 \mathrm{mV})$ est proportionnelle, à mieux de $1 \%$ près, au déplacement angulaire du pendule.

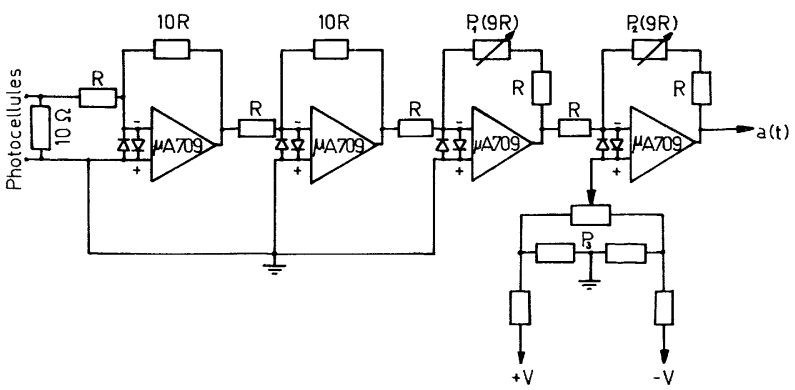

FIG. 5. - Conversion en tension du courant fourni par les photocellules et amplification de celle-ci.

Un amplificateur dont le gain peut varier de $10^{2}$ à $10^{4}$ amplifie cette tension. Cet amplificateur est composé de quatre amplificateurs opérationnels $\mu$ A709. Deux potentiomètres $P_{1}$ et $P_{2}$ permettent de régler le gain des deux derniers étages.

La tension de décalage de l'amplificateur ramenée à l'entrée peut être compensée au moyen du circuit $\mathrm{P}_{3}$.

3.1.3 Le déphaseur. - Il se compose essentiellement du circuit de déphasage, résistance ajustable $\mathrm{R}$ et condensateur $\mathrm{C}$, suivi d'un étage de puissance (Fig. 6). En modifiant cette résistance on peut ajuster le déphasage du signal $a(t)$ à la valeur $\pi / 2$ pour des fréquences comprises entre $10^{-2} \mathrm{c} / \mathrm{s}$ et $10 \mathrm{c} / \mathrm{s}$. Afin de rendre minimum la dérive thermique du montage on ajuste le courant drain du transistor à effet de champ à l'aide de la résistance réglable $R_{1}$. L'écrêtage du signal déphasé $\dot{a}(t)$ s'effectue par un comparateur du type $\mu$ A710. A l'aide de deux monostables $\mathbf{M}_{1}$ et $\mathbf{M}_{2}$ on forme des impulsions $c$.

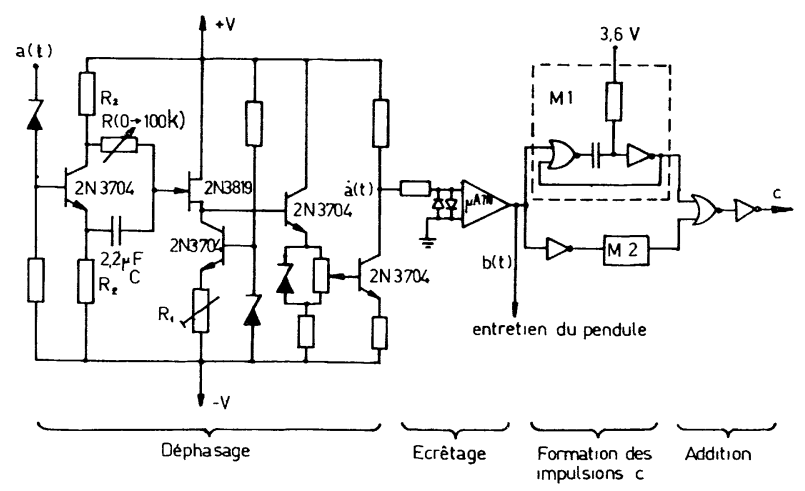

Fig. 6. - Circuit électronique pour la fabrication des impulsions $c: \mathrm{M}_{1,2}$, monostables.

3.2 Mesure digitale de la PÉRIOde DES OSCILlaTIONS DU PENDULE. - 3.2.1 Principe. - Nous mesurons le temps qui s'écoule pendant $n$ périodes d'oscillations du pendule. Le périodemètre fonctionne donc comme une porte électronique qui laisse passer pendant $n$ périodes des impulsions fournies par un oscillateur à quartz de fréquences $1 \mathrm{kc} / \mathrm{s}, 10 \mathrm{kc} / \mathrm{s}, 100 \mathrm{kc} / \mathrm{s}$ ou $1 \mathrm{Mc} / \mathrm{s}$. Afin de diminuer l'indétermination à l'enclenchement et au déclenchement du périodemètre, il est nécessaire de prendre $n$ aussi grand que possible.

Pour éviter de prolonger les cycles de mesures nous avons choisi de mesurer simultanément la période et les amplitudes $A_{i}$. Pour ce faire, nous accumulons au cours d'un cycle de mesure les impulsions délivrées par le périodemètre dans une mémoire temporaire. Ce n'est qu'à la fin du cycle considéré que le contenu de la mémoire est transféré dans le compteur du voltmètre digital, puis perforé sur la même bande que les mesures d'amplitude et de la température.

3.2.2 Le périodemètre. - Il est formé de deux portes électroniques $\mathrm{P}_{1}$ et $\mathrm{P}_{2}$ et de la mémoire temporaire $M$ (Fig. 7). Celle-ci est composée d'un compteur bi-directionnel [4] et d'un circuit qui détecte l'état zéro de ce compteur et fournit alors l'impulsion $z$.

L'ordre de mesure de la période est donné par une impulsion $p_{1}$ synchronisée avec le passage à zéro du signal $a(t)$. Dès cet instant, le signal horloge $s$ fourni par l'oscillateur à quartz, parvient dans la mémoire temporaire $M$ par l'intermédiaire de la porte $P_{1}$. Une impulsion $p_{2}$ bloque cette porte après $n$ périodes d'oscillations du pendule.

Le signal $f$ qui sert également d'ordre d'excitation des oscillations du pendule, inverse le sens de comptage de $\mathrm{M}$ et ouvre la porte $\mathrm{P}_{2}$. Par celle-ci, l'oscillateur à quartz débite un signal de $1 \mathrm{Mc} / \mathrm{s}$, à la fois 


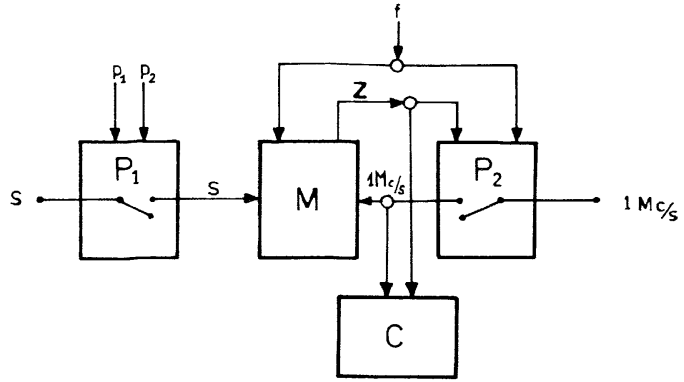

FIG. 7. - Synoptique du périodemètre : $\mathrm{P}_{2}$, porte pour le transfert du contenu de la mémoire temporaire $M ; P_{1}$, porte pour la mesure de la période; $\mathbf{C}$, compteur du voltmètre digital; $s$, signal horloge ; $p_{1,2}$, début et fin de la mesure de la période; $f$, commande du transfert du contenu de $\mathrm{M}$ dans $\mathrm{C}$; $z$, état zéro de $\mathbf{M}$ (fin du transfert).

dans $\mathrm{M}$ et dans le compteur $\mathrm{C}$ du voltmètre digital, et ceci jusqu'à l'annulation du contenu de $\mathrm{M}$, l'impulsion $z$ bloquant alors la porte $\mathrm{P}_{2}$. Ainsi le contenu du compteur $\mathrm{C}$ est égal au nombre d'impulsions de $s$ mémorisées pendant les $n$ oscillations du pendule. L'impulsion $z$ par la même occasion ordonne la perforation du contenu du compteur C.

Comme on peut le voir sur la figure 8 , la porte $P_{1}$ se compose de deux flip-flop $\left(\mathrm{F}_{3}\right.$ et $\left.\mathrm{F}_{4}\right)$ bloquant ou débloquant une porte $\operatorname{NOR}\left(\mathrm{N}_{1}\right)$ et par conséquent le signal horloge $s$. La porte $\mathrm{P}_{2}$ comprend un flip-flop $\left(\mathrm{F}_{2}\right)$

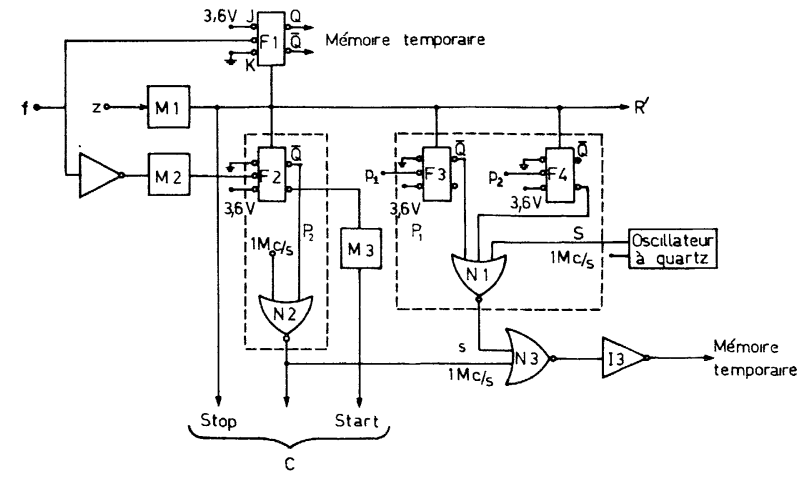

FIG. 8. - Le périodemètre: $\mathrm{P}_{1,2}$, portes (voir Fig. 7); $\mathbf{M}_{1,2}$, monostables; $s$, signal horloge ; $\mathrm{R}^{\prime}$, remise à zéro de la mémoire temporaire; $\mathrm{C}$, compteur du voltmètre digital.

et une porte $\mathrm{NOR}\left(\mathrm{N}_{2}\right)$ bloquant ou débloquant le signal de $1 \mathrm{Mc} / \mathrm{s}$. Le signal $s$ et le signal de $1 \mathrm{Mc} / \mathrm{s}$ sont sommés à l'aide des éléments $\mathrm{N}_{3}$ et $\mathrm{I}_{3}$. Le sens de comptage de $\mathrm{M}$ est déteıminé par le flip-flop $\mathrm{F}_{1}$.

3.3 Mesure digitale de la température. - La température de l'échantillon est mesurée à l'aide d'une résistance de platine placée en contact avec la chape du pendule (Fig. 1). Afin de mesurer sa résistance, on y fait passer un courant constant et l'on mesure la tension $V_{\mathrm{T}}$ aux bornes de celle-ci. La mesure de cette tension, après amplification [3] est effectuée par le même convertisseur analogique-digital que celui qui sert à la mesure de l'amplitude des oscillations du pendule. La commutation des deux signaux $a(t)$ (ampli- tude) et $V_{\mathrm{T}}$ (température) sur le convertisseur se fait au moyen d'une porte électronique (Fig. 9) commandée par l'impulsion $c$ pour la mesure de $A_{i}$ et par l'impulsion $e$ pour la mesure de $V_{\mathbf{T}}$.

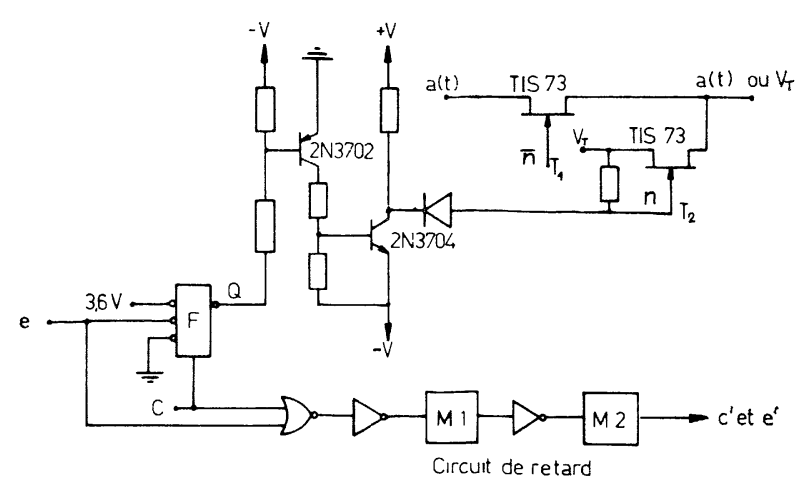

FIG. 9. - Porte électronique : $a(t)$, élongation du pendule; $V_{\mathrm{T}}$, température de l'échantillon; $e$, débloquage de $V_{\mathrm{T}}$ et bloquage de $a(t) ; c$, opération inverse; $\mathbf{M}_{1,2}$, monostables.

L'impulsion de commande e de mesure de la température fera basculer le flip-flop $(\mathrm{F})$ et par conséquent rendra conducteur le transistor $T_{2}$ et bloquera le transistor $T_{1}$. Légèrement retardé par le circuit de retard, le signal $e^{\prime}$ parviendra au convertisseur analogique-digital et déclenchera la mesure de $V_{\mathrm{T}}$.

3.4 CORRECTION DE L'eFFet DE LA DÉRIVE MÉCANIQUE DU PENDULE. - On n'intervient pas à proprement parler sur la dérive mécanique du pendule, mais on centre le spot lumineux sur l'écran des photocellules, au moyen d'un miroir mobile orienté par un servomoteur (Fig. 1). L'erreur de centrage $\varepsilon$ est donnée par un filtre passe-bas (Fig. 10) qui restitue la composante

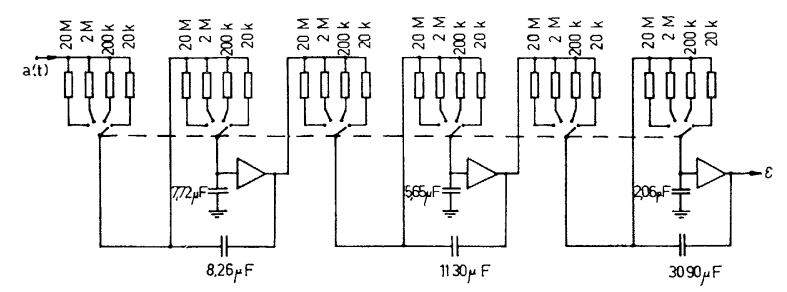

Fig. 10. - Détection de la dérive mécanique du pendule.

continue du signal $a(t)$. Le signal d'erreur $\varepsilon$ est ensuite dirigé sur un détecteur à deux seuils (positif et négatif) qui commande le servo-moteur.

Le filtre passe-bas est un filtre actif à 3 étages [5]. Pour avoir différentes fréquences de coupure $(0,001 \mathrm{c} / \mathrm{s}$, $0,01 \mathrm{c} / \mathrm{s}, 0,1 \mathrm{c} / \mathrm{s}$ ou $1 \mathrm{c} / \mathrm{s})$, on peut au moyen d'un commutateur, modifier les résistances intervenant dans les circuits du filtre.

Etant donné la valeur très élevée de ces résistances, l'impédance d'entrée des amplificateurs à gain unité doit également être très grande. Ceci a été réalisé à l'aide du montage de la figure 11 . Une résistance ajustable $\mathrm{R}$ permet de varier le courant drain du transistor à effet de champ de sorte que la dérive thermique de 


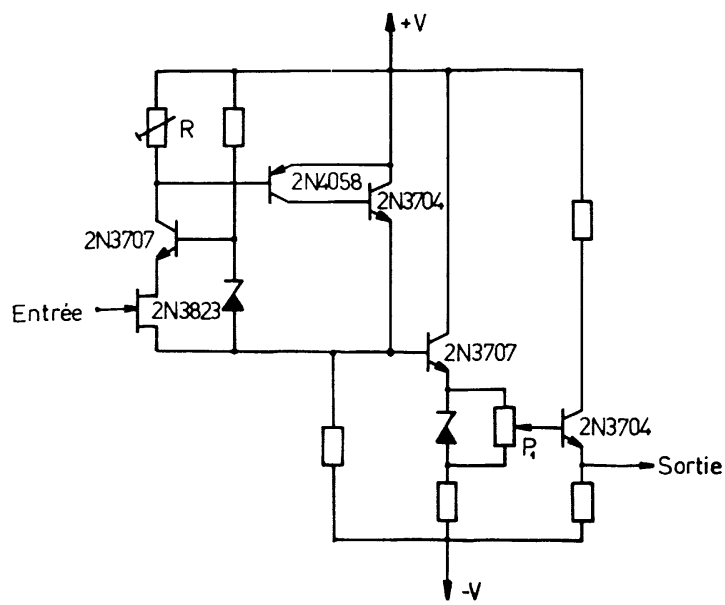

Fig. 11. - Amplificateur à gain unité.

l'amplificateur puisse être rendue minimum. A l'aide $\mathrm{du}$ potentiomètre $\mathrm{P}_{1}$ on peut encore ajuster la tension de sortie de l'amplificateur sur celle de l'entrée.

La tension $\varepsilon$ correspondant à la dérive mécanique du pendule est comparée à 2 valeurs $\pm \varepsilon_{0}$ par le dispositif électronique qui commande le servo-moteur. Ce dispositif est formé d'un amplificateur (gain 10) suivi de deux triggers (Fig. 12). Les signaux fournis par ces

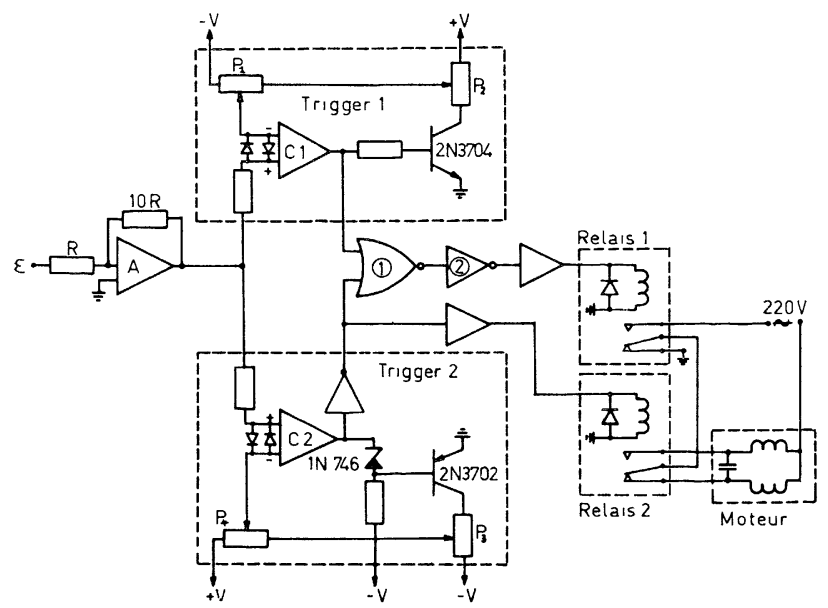

Fig. 12. - Correction de l'effet de la dérive mécanique du pendule: $\mathbf{C}_{1,2}$, comparateur $\mu \mathrm{A} 710$; A, amplificateur opérationnel $\mu \mathrm{A} 709 ; \varepsilon$, dérive mécanique du pendule.

triggers sont sommés (composants 1 et 2) et commandent le relais 1 qui actionne le moteur. Le relais 2 qui détermine le sens de la correction est commandé par le trigger 2. Pour chacun des deux triggers on peut ajuster les deux tensions de basculement à l'aide des potentiomètres $\mathrm{P}_{1}$ et $\mathrm{P}_{2}$, respectivement $\mathrm{P}_{3}$ et $\mathrm{P}_{4}$. Il suffit pour ceci que le courant qui traverse $\mathrm{P}_{2}$ et $\mathrm{P}_{3}$ soit beaucoup plus grand que le courant qui passe par $\mathrm{P}_{1}$ et $\mathrm{P}_{4}$.

3.5 Calcul analogiQue du fRotTement intéRIEUR. - 3.1.5 Le principe. - Il est utile de connaître le frottement intérieur au cours de l'expérience, sans attendre les résultats numériques donnés par l'ordinateur. Nous avons donc développé un dispositif qui calcule et enregistre le frottement intérieur sous forme graphique. Ce dispositif se base sur la formule (1). C'est-à-dire, il mesure les tensions $A_{i}$ et $A_{i+n}$, et soustrait le logarithme de $A_{i+n}$ du logarithme de $A_{i}$.

Les tensions $A_{i}$ et $A_{i+n}$ ne peuvent être mesurées en même temps. On est ainsi obligé de mémoriser les quatre grandeurs $A_{i}^{+}, A_{i}^{-}, A_{i+n}^{+}$et $A_{i+n}^{-}$, et le frottement intérieur qui en sera déduit ne pourra être calculé qu'au moment où ces quatre grandeurs seront connues. Pour la continuité de l'enregistrement, la valeur du frottement intérieur est introduite dans une mémoire supplémentaire connectée à l'enregistreur.

Le synoptique du calculateur est représenté sur la figure 13. Les opérations qui doivent être effectuées dans un ordre chronologique donné, sont commandées par une unité logique. Cette logique reçoit les impulsions $c$ (qui correspondent aux différents extrêma de $a(t))$, ainsi que les impulsions de mise à zéro $\mathrm{R}$ à chaque lancement du pendule. Ces dernières synchronisent le calculateur afin que le calcul analogique du frottement intérieur se fasse entre deux lancements du pendule. La logique fournit les ordres de mémorisation $l_{i}^{+}, l_{i}^{-}, l_{i+n}^{+}$et $l_{i+n}^{-}$à partir de la succession des impulsions $c$. Lorsque la dernière valeur $A_{i+n}^{-}$est mémorisée et que par conséquent la valeur du frottement intérieur est connue, une impulsion $g$ ordonne à la mémoire $\mathrm{M}_{5}$ de la mémoriser.

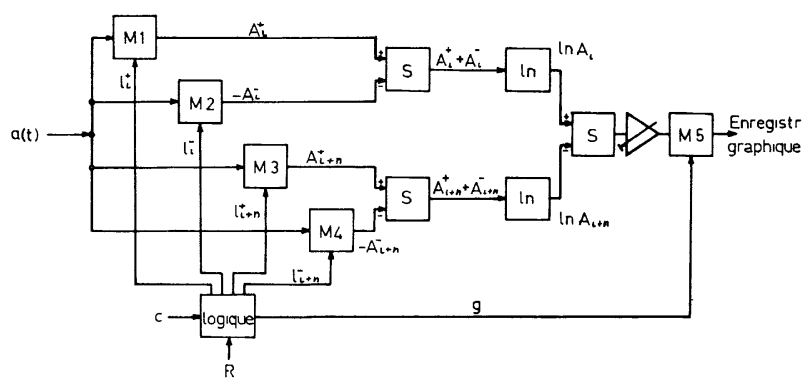

Fig. 13. - Synoptique du calculateur du frottement intérieur: $\mathbf{M}_{1,2,3,4,5}$, mémoires analogiques; $s$, soustracteur ; c, impulsions horloge $; l_{i}^{+}, l_{i}^{-}, l_{i+n}^{+}, l_{i+n}^{-}$et $g$, commandes des mémoires analogiques; $\mathrm{R}$, synchronisation du calculateur.

\subsubsection{Les mémoires et le calcul du logarithme. -} Lorsqu'une impulsion $l$ (l'une des $l_{i}^{+}$, etc.) est délivrée par la logique du calculateur, le transistor $T$ est rendu conducteur (Fig. 14). A ce moment la mémoire est en fait un amplificateur à gain 1 , de sorte que la tension de sortie soit égale à la tension d'entrée. A la fin de l'impulsion $l$ le transistor se trouve de nouveau bloqué, mais étant donné que le condensateur $\mathrm{C}$ ne peut pratiquement plus se décharger, la tension est conservée sur la sortie.

Etant donné que cette mémorisation a lieu pendant un temps très court (env. $10 \mathrm{~ms}$ ) par rapport à la période de $a(t)$ et à l'instant où $a(t)$ passe par un extrêmum, la tension mémorisée est bien une valeur de crête de $a(t)$. Deux circuits limitateurs évitent que l'amplificateur opérationnel $\mu$ A709 ne se sature. Le 


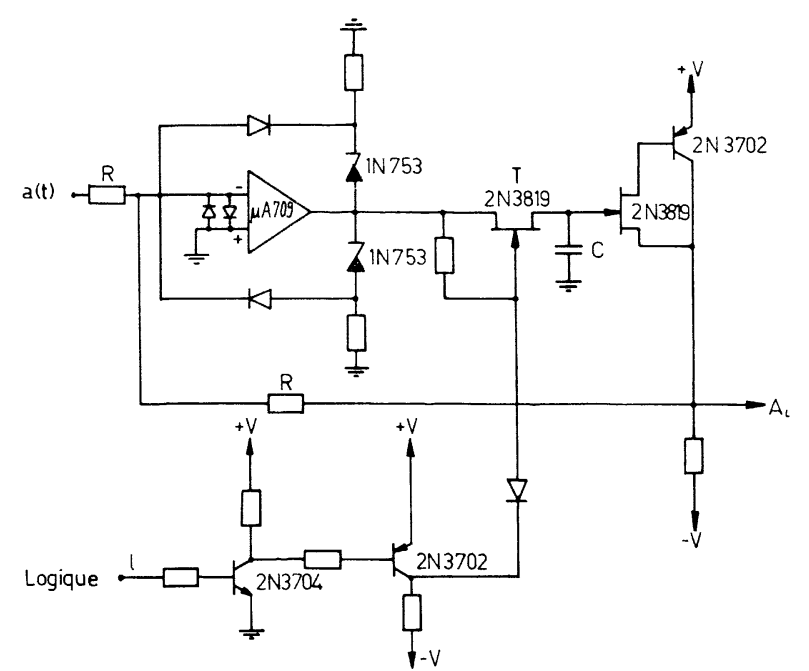

FIG. 14. - Mémoire analogique $: l$, commande de la mémorisation.

logarithme est calculé au moyen de transducteurs logarithmiques [6].

3.6 Courant D'EXCITATION POUR L'ENTRETIEN DES OSCILlations DU PENDULE. - Lorsqu'un cycle de mesures est achevé on relance le pendule à une amplitude donnée au moyen de deux solénoïdes qui exercent une force alternée sur deux baguettes de ferrite fixées au balancier du pendule. Le courant d'excitation est obtenu en appliquant la tension $b(t)$ (Fig. 6) aux bornes des solénoïdes.

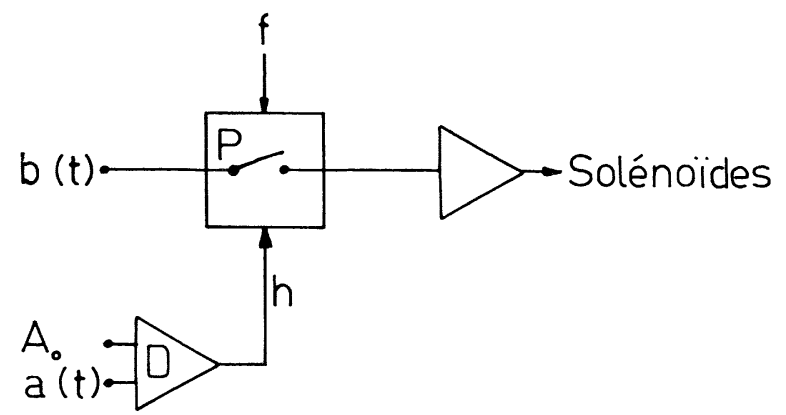

FIG. 15. - Excitation des solénoïdes $\mathrm{P}$, porte électronique ; $\mathrm{D}$, comparateur ; $\mathrm{A}_{0}$, valeur de consigne ; $f$, début de l'excitation ; $h$, fin de l'excitation.

Dès que l'amplitude du signal $a(t)$ atteint une valeur de consigne $A_{0}$, la porte électronique $\mathrm{P}$ (Fig. 15) coupe $b(t)$. A cet effet on compare la valeur de crête de $a(t)$ à la valeur de consigne $A_{0}$ au moyen du comparateur D. Ce dernier ferme au moyen d'un signal $h$ la porte électronique $\mathrm{P}$ et bloque par conséquent le signal d'excitation $b(t)$. L'excitation se fait à nouveau dès que cette porte est ouverte par le signal $f$.

3.7 COMMANDE DES DIVERSES OPÉRATIONS DE MESURE, DE LA PERFORATION DE CELLES-CI, AINSI QUE DE L'ENTRETIEN DU PENDULE. - Les opérations de mesure et l'excitation des oscillations du pendule sont commandées et contrôlées par une logique centrale. Elle est synchronisée avec les oscillations du pendule à l'aide du signal $a(t)$. Elle contrôle ou délivre les impulsions qui déclenchent les différentes opérations au cours d'un cycle de mesures, c'est-à-dire :

- Les impulsions $c$ pour la mesure des valeurs de crête de $a(t)$. En fait, ces impulsions proviennent du déphaseur (3.1.3) et la logique centrale ne fait que bloquer ces impulsions pendant $n-1$ périodes afin que la mesure des $A_{i}^{+}$et $A_{i}^{-}$ne se fasse que toutes les $n$ périodes.

- Les impulsions $d$ pour la perforation des résultats. Elles sont fournies par le convertisseur analogiquedigital à la fin de la conversion d'une grandeur analogique. Lors de la mesure des extrêma de $a(t)$ la logique centrale bloque les impulsions $d$ consécutives à la mesure des $A_{i}^{+}$pour ne laisser passer que les impulsions qui suivent la mesure des $A_{i}^{-}$, de sorte que l'addition de ces deux grandeurs se fasse directement par le compteur du voltmètre digital.

- Le signal $f$ qui enclenche le courant d'excitation des oscillations du pendule.

- Les impulsions $p_{1}$ et $p_{2}$ pour le début et la fin de la mesure de la période des oscillations du pendule.

- L'impulsion de mise à zéro $\mathrm{R}$ destinée à mettre à l'état zéro les flip-flop de la logique centrale et de la logique du calculateur analogique $d u$ frottement intérieur.

- Une impulsion $e$ pour la mesure de la température.

4. Conclusions. - Le frottement intérieur calculé par ordinateur à partir de la mesure des valeurs de crête de $a(t)$ est déterminé avec une précision de $0,5 \%$. Le calcul analogique du frottement intérieur à l'aide du dispositif décrit dans 3.6 donne une précision de $2 \%$.

La fréquence des oscillations du pendule, qui peut être choisie entre $10^{-2} \mathrm{c} / \mathrm{s}$ et $10 \mathrm{c} / \mathrm{s}$, est mesurée avec une précision relative de $\pm 10^{-5}$.

Remerciements. - Les auteurs remercient Messieurs Grosjean et Falconnier pour leur aimable collaboration pour la construction du pendule de torsion et des appareils.

\section{Bibliographie}

[1] Zener, Elasticité et anélasticité des Métaux, Dunod, Paris, 1955.

[2] Martinet (B.), Convertisseur amplitude-numérique pour la mesure automatique du frottement intérieur au moyen d'un pendule de torsion, Neue Technik, 7, Edition B, № 24, 1965.

[3] Grandchamp (P. A.), Fornerod (R. C.), J. of Sci. Inst 1970, 3, 219.
[4] Micrologic Handbook, 1965, S. G. S., Fairchild, London.

[5] Bildstein (P.), Une méthode simple de conception et réalisation de filtres actifs, Electronique Industrielle, No 112, avril 1968.

[6] Application Manual of Operational Amplifier, Philbrick/Nexus Research, Nimrod Press, Boston, 1968. 